
\title{
28 Research Square \\ Effects of intraoperative hypotension on postoperative renal function in arthroscopic shoulder surgery: a retrospective study
}

Chaeseong Lim

Chungnam National University College of Medicine https://orcid.org/0000-0002-2356-8999

\section{Seounghun Lee}

Chungnam National University Hospital

Woosuk Chung

Chungnam National University

Hoseop Kim

Chungnam National University Hospital

\section{Seungbin Jeon}

Chungnam National University Hospital

Yoon Hee Kim ( $\nabla$ yhkim040404@gmail.com )

\section{Research article}

Keywords: beach chair position; creatinine; hypotension; surgery

Posted Date: March 23rd, 2020

DOI: https://doi.org/10.21203/rs.3.rs-18194/v1

License: (c) (i) This work is licensed under a Creative Commons Attribution 4.0 International License. Read Full License

Version of Record: A version of this preprint was published at Chirurgia on January 1st, 2022. See the published version at https://doi.org/10.23736/S0394-9508.20.05179-7. 


\section{Abstract}

Background: Arthroscopic shoulder surgery tends to cause a drop in blood pressure due to the beach chair position used during the procedure, including activation of the Bezold-Jarisch reflex. We hypothesized that patients with low blood pressure undergoing arthroscopic shoulder surgery in the beach chair position would also have reduced renal function after surgery.

Methods: The medical records of patients $(N=643)$ undergoing arthroscopic shoulder surgery in the beach chair position between July 2013 and May 2015 were examined. The vital signs were measured at 5-minute intervals, and the number of non-invasive blood pressure (NIBP) measurements in the upper arm dropping below a mean arterial pressure (MAP) of $50 \mathrm{mmHg}$ (MAP50) or $60 \mathrm{mmHg}$ (MAP60) were recorded. The primary outcome was change in creatinine immediately after surgery relative to the preoperative level (Cr post/pre). The factors affecting $\mathrm{Cr}$ post/pre were examined by correlation analysis.

Results: A total of 597 patients were included in the analysis. Longer duration of hypotension during surgery (MAP50) was correlated with higher $\mathrm{Cr}$ post/pre $(R=0.107, \mathrm{P}=0.010)$. However, the correlation between MAP60 and $\mathrm{Cr}$ post/pre was not significant $(R=0.033, P=0.431)$.

Conclusions: It is necessary to ensure that the MAP does not fall below $50 \mathrm{mmHg}$ to ensure sufficient renal function during general anesthesia for shoulder arthroscopy in a beach chair position.

\section{Background}

Intraoperative hypotension has been reported to reduce postoperative kidney function, which can cause acute kidney injury (AKI) during the perioperative period. The term "acute kidney injury", which has replaced the term "acute renal failure", includes both renal malfunction and tissue damage [1]. AKI affects morbidity and mortality in both the short and long term, increasing the risk of chronic and terminal kidney diseases [2,3]. There are no definitive criteria for treating hypotension during surgery, but it has been reported to be related to AKI when the mean arterial pressure (MAP) is below $55-60 \mathrm{mmHg}[4,5]$.

Arthroscopic shoulder surgery tends to cause a drop in blood pressure due to the beach chair position, including activation of the Bezold-Jarisch reflex [6]. Significant hypotension can lead to cerebral hypoperfusion and neurological complications, such as cerebral ischemia, pituitary apoplexy, and cardiovascular collapse[7-9]. However, the kidneys are located below the heart when in the beach chair position, and it is assumed that renal blood flow can be maintained relatively easily compared to the blood pressure (as measured at the upper arm) due to gravity. In this study, cases where the MAP fell below $50-60 \mathrm{mmHg}$ according to the anesthesia records were examined. We performed this retrospective study to determine whether patients with low blood pressure undergoing arthroscopic shoulder surgery also had reduced renal function after surgery. It is hypothesized that creatinine will increase after surgery in patients whose MAP has dropped below $50 \mathrm{mmHg}$ during surgery.

\section{Methods}




\section{Study setting}

This study was approved by the institutional review board of Chungnam National University Hospital (approval number: CNUH 2018-09-029) and consent was waived. The medical records of patients $(N=$ 643) who underwent arthroscopic shoulder surgery in the beach chair position between July 2013 and May 2015 were reviewed. The vital signs were recorded at 5-minute intervals in the anesthesia records, along with the number of non-invasive blood pressure (NIBP) measurements in the upper arm that dropped below the MAP of 50-60 mmHg. Patients whose MAP fell below $50 \mathrm{mmHg}$ even once were classified into the hypotension group (Group 1), while those in whom blood pressure did not fall below 50 $\mathrm{mmHg}$ were classified into the normal group (Group 2). Renal function was examined before, immediately after, 1 day after, and 3 days after surgery. Blood tests were performed immediately after surgery in most patients, but many patients did not undergo blood tests 1 or 3 days after surgery.

\section{Participants}

After collecting the medical information of patients who received arthroscopic rotator cuff repair surgery $(N=643)$ in a beach chair position between July 2013 and May 2015, the following exclusion criteria were applied: no anesthesia records $(n=1)$, or no blood test results before $(n=1)$ or immediately after $(n$ $=41$ ) surgery. Finally, a total of 597 patients (Fig. 1) were included in the analysis after excluding three additional patients with renal failure who showed a preoperative glomerular filtration rate (GFR) $\leq 15$ $\mathrm{mL} / \mathrm{min} / 1.73 \mathrm{~m}^{2}$.

\section{Anesthetic technique}

In most patients, anesthesia was induced with propofol at $1-2 \mathrm{mg} / \mathrm{kg}$ and rocuronium at $0.6-1.0 \mathrm{mg} / \mathrm{kg}$. Anesthesia was maintained with sevoflurane or desflurane, and sustained thereafter with remifentanil at $150-350 \mu \mathrm{g} / \mathrm{h}$. Before induction of general anesthesia, $15 \mathrm{cc}$ of $1 \%$ lidocaine and $15 \mathrm{cc}$ of $0.375 \%$ ropivacaine were administered for interscalene block under ultrasound guidance.

\section{Primary outcome}

The primary outcome was the change in creatinine $(\mathrm{Cr})$ level immediately after surgery compared to the preoperative $\mathrm{Cr}$ level (Cr post/pre).

\section{Secondary outcome}

Data were compared between the groups 1 and 3 days after surgery. Patients without blood test results (postoperative day [POD]1, POD3) were excluded from the analysis on the basis of missing data. The factors associated with $\mathrm{Cr}$ post/pre were analyzed. In all patients, the correlations of the MAP50 (number 
of times the MAP was recorded as $<50 \mathrm{mmHg}$ ) with all factors of interest were assessed to determine the factors associated with significantly decreased blood pressure during surgery. To determine the level of hypotension that is safe for the kidney, partial correlation analysis of $\mathrm{Cr}$ post/pre with MAP60 (number of times the MAP was recorded as $<60 \mathrm{mmHg}$ ) was also performed.

\section{Statistics}

\section{Sample size}

In a previous paper[10], 278 patients were enrolled for a study to prove the importance of serum $\mathrm{Cr}$ as a test to evaluate postoperative function. In the calculation using $G^{*}$ Power (version 3.1, Franz Faul \& Edgar Erdfelder, Trier, Germany), a total of 210 of 105 people in each group could obtain an effect size of 0.5 , an alpha error of 0.05 and a power of $95 \%$. Since the number of arthroscopic rotator cuff repair operations per year was about 200 in our center, it was estimated that an anesthesia record review of nearly two years would be sufficient. What was more important was to collect only data from the same surgeon who underwent surgery in the same position under the same diagnosis. The study period was clearly defined as the period from the opening of our joint center in July 2013 to the resignation in May 2015 of the single orthopedic surgeon specializing in shoulders.

\section{Demographic data}

Demographic data (Table 1), and the Cr post/pre, were compared between Groups 1 and 2 using the independent samples $t$ test (Table 2).

\section{Correlation analysis}

Spearman rho nonparametric correlation analysis was performed to determine the factors associated with renal function, i.e., the $\mathrm{Cr}$ post/pre, after surgery. The MAP50 was included as a measure of the duration of hypotension, and correlations thereof with all factors of interest were calculated. The variables included in the correlation analysis were as follows: total amount of intravenous fluid administered, age, height, weight, American Society of Anesthesiologists (ASA) physical status, history of hypertension, history of diabetes mellitus, sex, MAP50, Cr post/pre, preoperative blood urea nitrogen (BUN), preoperative $\mathrm{Cr}$, and preoperative GFR. Statistically significant variables in the correlation analysis were used as control variables in the partial correlation analysis, of MAP60 with $\mathrm{Cr}$ post/pre.

Statistical analyses were performed using IBM SPSS Statistics 24 (IBM Corp., Armonk, NY, USA).

\section{Results}

\section{Comparison of demographic data}


The demographic data of the two groups are shown in Table 1. All variables except weight and diabetes were significantly different between the two groups. The proportions of patients who were elderly, female, of small stature, in a high ASA class, and hypertensive were all higher in Group 1 than Group 2.

The results of the group comparison are shown in Table 2. There were significant group differences in the preoperative BUN level $(15.90 \pm 4.49$ vs. $14.98 \pm 3.99, P=0.021)$ and $\mathrm{Cr}$ level $(0.74 \pm 0.18$ vs. $0.79 \pm 0.19$, $P=0.008)$, and in the GFR (109.72 \pm 25.44 vs. $116.88 \pm 37.34$, respectively, $P=0.034)$ immediately after surgery.

\section{Correlation analysis}

Spearman rho correlation analysis was performed to determine the factors associated with renal function (Cr post/pre) after surgery, and the results are shown in Table 3. Higher body weight $(\mathrm{R}=0.145, P=$ $0.000)$, longer duration of hypotension (MAP50) during surgery $(R=0.107, P=0.010)$, the administration of less intravenous fluid during surgery $(\mathrm{R}=-0.081, P=0.049)$, and higher preoperative GFR $(\mathrm{R}=0.208$, $P=0.000$ ) were associated with $\mathrm{Cr}$ post/pre. The partial correlation between MAP60 and $\mathrm{Cr}$ post/pre was not significant $(\mathrm{R}=0.033, P=0.431)$. The correlations of MAP50 with all factors of interest are summarized in Table 4. Greater intravenous fluid administration during surgery $(R=0.205, P=0.000)$, lower preoperative GFR $(R=-0.114, P=0.006)$, and lower $\operatorname{Cr}(R=-0.104, P=0.012)$ were associated with MAP50 (i.e., longer duration of intraoperative hypotension). However, it should be noted that the anesthesiologist increased intravenous fluid administration because the blood pressure continued to drop.

\section{Case analysis}

Among 597 patients, the postoperative plasma Cr level showed an increase of $>25 \%$ in 12 cases, $>50 \%$ in four cases, and $>100 \%$ in one case. One patient (a 43-year-old woman) in whom the $\mathrm{Cr}$ level more than doubled postoperatively was healthy (ASA class I), showing no underlying disease and no occasion on which the MAP dropped below $60 \mathrm{mmHg}$. However, the $\mathrm{Cr}$ level increased by 237\%, from 0.6 to 1.42 $\mathrm{mg} / \mathrm{dL}$, because only $250 \mathrm{~mL}$ of plasmalyte was administered intravenously during the 100-minute surgery. This volume of fluid was quite low given that the patient weighed $67 \mathrm{~kg}$, where the average amount of fluid administered in her group was $624 \mathrm{~mL}$. Fortunately, after 1 day, the $\mathrm{Cr}$ level dropped to $0.39 \mathrm{mg} / \mathrm{dL}$, which was lower than that before surgery.

\section{Discussion}

In this study, renal function tended to improve with a decrease in the Cr level and increase in the GFR after surgery. As shown in Table 2, the GFR of Group 2 increased immediately after surgery compared to that of Group 1, in accordance with longer-duration low blood pressure. Table 3 shows that longer-duration low blood pressure was associated with a higher $\mathrm{Cr}$ post/pre immediately after surgery. Taken together, 
these observations indicated that renal function improved after shoulder arthroscopy in the beach chair position, but the degree of improvement appeared to be lower in patients experiencing periods of hypotension, i.e., a MAP $<50 \mathrm{mmHg}$.

General anesthesia inhibits the lower extremity muscle contraction necessary for maintenance of venous return to the heart. Venous congestion caused by the beach chair position during shoulder surgery under general anesthesia decreases preload and promotes abnormal cardiac contractions due to catecholamine hypersecretion [6]. The surgeon should ask the anesthesiologist to lower the patient's blood pressure to improve arthroscopic vision. Blood pressure naturally drops after general anesthesia in the beach chair posture. Therefore, if the operation is performed under general anesthesia, large amounts of vasopressors are needed, and if the operation is performed only with interscalene block and sedation, large amounts of blood pressure-lowering agents, such as nicardipine, are needed [11]. These observations raise questions regarding the appropriate blood pressure for shoulder arthroscopy when the patient is in the beach chair posture. In a retrospective study of 5,127 patients undergoing noncardiac surgery, an increased risk of postoperative AKI (defined as a $>50 \%$ or $>0.3 \mathrm{mg} / \mathrm{dL}$ increase in serum $\mathrm{Cr}$ concentration) was found in cases with intraoperative MAP $<60 \mathrm{mmHg}$ for $>20$ minutes and $<55 \mathrm{mmHg}$ for $>10$ minutes [4]. In the present study, MAP50 was significantly correlated with $\mathrm{Cr}$ post/pre, while no such correlation was observed for MAP60. Therefore, taking postoperative renal function into consideration, it is preferable to maintain the MAP above $60 \mathrm{mmHg}$ during shoulder arthroscopic surgery with the patient in the beach chair position.

In a study of high risk patients undergoing cardiac surgery, postoperative renal function deterioration was defined as an increase in the serum $\mathrm{Cr}$ level during the first postoperative week of $\geq 25 \%$ compared to the preoperative level [12]. GFR, defined as the amount of blood the kidney cleans in 1 minute, is the most important measure of kidney function and normal values are in the range $90-120 \mathrm{~mL} / \mathrm{min}$. That is, the kidneys in subjects with normal kidney function can clean $90-120 \mathrm{~mL}$ of blood in 1 minute, which translates to about 120-180 L of blood filtered throughout the day. Kidney function is usually assessed using the Modification of Diet in Renal Disease (MDRD) equation, which calculates the GFR.

The MDRD equation has been studied in thousands of white and black kidney disease patients in the USA, making it easy to assess the GFR based on age, gender, race, and serum $\mathrm{Cr}$ concentration. This method has the advantage of easy calculation of GFR without the need for complicated procedures such as urine collection or nuclear medicine tests. However, as the formula is based on patients with kidney disease, it tends to slightly underestimate the values in people with early kidney disease, and in healthy people with GFR $\geq 60 \mathrm{~mL} / \mathrm{min}$. In addition, as these studies were performed in white and black subjects, there is some controversy about the applicability to Asian races, such as Koreans. Therefore, the $\mathrm{Cr}$ post/pre was set as the primary outcome in this study. As shown in Table 2, follow-up results more than 1 day after the operation were not available in many patients, and all such indicators were therefore excluded from the correlation analysis. There was a significant difference in GFR between Groups 1 and 2 $(109.72 \pm 25.44$ vs. $116.88 \pm 37.34$, respectively, $P=0.034)$ immediately after surgery. However, as the 
demographic data of the two groups were very different (Table 1), the validity of the comparison is not clear.

In fact, there have been many reports of complications related to brain function associated with surgery in the beach chair position. Significant hypotension can lead to cerebral hypoperfusion and neurological complications, such as cerebral ischemia, pituitary apoplexy, and cardiovascular collapse [7-9]. To prevent these complications, some studies used sequential compression devices [13] or attempted intravenous bolus administration of arginine vasopressin [14]. In the present study, when blood pressure was low, $50 \propto \mathrm{g}$ of phenylephrine or $5 \mathrm{mg}$ of ephedrine was injected as a bolus. At the same time, 200$300 \mathrm{cc}$ of plasmalyte was loaded so that the medication could exert its effects quickly and correct the volume depletion. If the vasopressor requirement is high, sustained phenylephrine infusion $(1-2 \mathrm{~g} / \mathrm{h})$ should be started. However, because most patients had an intravenous 18-gauge cannula in the arm opposite the surgical shoulder, NIBP was difficult to acquire every 5 minutes. Although anti-reflux valves were used in all patients, reflux or blockage nevertheless occurs frequently. Therefore, patients with wellmaintained blood pressure may receive only low levels of fluid. This is thought to account for why only $250 \mathrm{cc}$ of fluid was administered to the patient in whom the $\mathrm{Cr}$ level more than doubled. One study showed that patients undergoing shoulder surgery in the beach chair position are more likely to show impaired autoregulation, as demonstrated by the higher cerebral oximetry index and lower regional cerebral oxygen saturation $\left(\mathrm{rScO}_{2}\right)$ than patients undergoing surgery in the lateral decubitus position [15]. Subtracting $1.35 \mathrm{mmHg}$ from the blood pressure measured in the arm or leg for each $1 \mathrm{~cm}$ of head elevation has been recommended to avoid cerebral hypoperfusion due to the effect of gravity. However, there have been no previous studies of the function of the kidney in the beach chair position, when it is below the heart, or on the regulation of blood flow to the kidney. Two mechanisms have been shown to be involved in renal autoregulation, i.e., the myogenic response and the tubuloglomerular feedback mechanism [16]. A piglet study [17] concluded that renovascular autoregulation is impaired in advance of cerebrovascular autoregulation during hemorrhagic shock. Taken together, these findings indicate cerebral blood flow control is more difficult than renal blood flow control in the beach chair position, so care is needed. Reflecting the results of this study, it is sufficient to monitor brain function and $\mathrm{rScO}_{2}$ in cases with MAP $\geq 60 \mathrm{mmHg}$, but renal damage may occur in cases with MAP $\leq 50 \mathrm{mmHg}$. Therefore, it is necessary to prevent the MAP from falling below $50 \mathrm{mmHg}$, and if it does drop below this level, active treatment to increase the blood pressure immediately is necessary.

This study had some limitations. First, it used a retrospective design, and consistent and accurate administration of fluids and vasoactive drugs may not have been achieved. However, the same surgeon performed the same operations, and the anesthesia team used the same guidelines. Second, the number of patients was different between the hypotension group (Group 1) and control group (Group 2), and there were differences in the demographic data; thus we performed correlation analysis. Third, there may be a measurement accuracy problem. It is not possible to know how accurate it is because the pressure is not measured directly through a catheter, but measured by NIBP. However, the non-surgical arm was fixed to a dedicated armrest, so the measurement was not interfered with by the surgeon or assistant. Renal blood 
flow and pressure in humans cannot yet be measured directly; new medical devices and equipment or research techniques are thus required [18].

\section{Conclusions}

The number of MAP measurements $<50 \mathrm{mmHg}$ showed an association with the plasma Cr level after surgery. On the other hand, as the number of MAP measurements $<60 \mathrm{mmHg}$ was not correlated with $\mathrm{Cr}$ post/pre. It is necessary to ensure that the MAP does not fall below $50 \mathrm{mmHg}$ to ensure sufficient renal function during general anesthesia for shoulder arthroscopy in a beach chair position. In addition to blood pressure, body weight and the amount of intravenous fluid administered were also associated with Cr post/pre. Randomized controlled trials are needed to measure renal blood flow in real-time using ultrasound.

\section{Abbreviations}

NIBP

Non-invasive blood pressure

MAP

Mean arterial pressure

AKI

Acute kidney injury

IRB

Institutional review board

GFR

Glomerular filtration rate

$\mathrm{Cr}$

Creatinine ( $\mathrm{Cr}$ )

POD

Postoperative day

ASA

American Society of Anesthesiologists

BUN

Blood urea nitrogen

MDRD

Modification of Diet in Renal Disease

$\mathrm{rScO}_{2}$

Regional cerebral oxygen saturation

\section{Declarations}


This study was approved by the institutional review board of Chungnam National University Hospital (approval number CNUH 2018-09-029) and consent was waived.

\section{Consent to publish}

Not applicable.

\section{Availability of data and materials}

The raw data of the current study are available from the corresponding author on request.

\section{Competing interests}

The authors declare no conflict of interest.

\section{Funding}

This work was supported by research fund of Chungnam National University (Award Number: 2019-054901). This fund contributed to the overall papers, including data collection and analysis.

\section{Authors' contributions}

Conceptualization, Seounghun Lee; Data curation, Seungbin Jeon; Formal analysis, Woo suk Chung; Software, Hoseop Kim; Writing - original draft, Chaeseong Lim; Writing - review \& editing, Yoon hee Kim. All authors have read and approved the manuscript.

\section{Acknowledgements}

These results were presented at the Euroanaesthesia 2019 (Vienna, Austria, 01/06/2019 - 03/06/2019) in electronic poster format. While analyzing the statistics in a new method, the abstracts that were presented have changed slightly. The English in this document has been checked by at least two professional editors, both native speakers of English. For a certificate, please see:

http://www.textcheck.com/certificate/MoAzdu

\section{References}

1. Makris K, Spanou L: Acute Kidney Injury: Definition, Pathophysiology and Clinical Phenotypes. Clin Biochem Rev 2016, 37(2):85-98.

2. Rimes-Stigare C, Frumento P, Bottai M, Martensson J, Martling CR, Walther SM, Karlstrom G, Bell M: Evolution of chronic renal impairment and long-term mortality after de novo acute kidney injury in the critically ill; a Swedish multi-centre cohort study. Crit Care 2015, 19:221.

3. Rimes-Stigare C, Frumento P, Bottai M, Martensson J, Martling CR, Bell M: Long-term mortality and risk factors for development of end-stage renal disease in critically ill patients with and without 
chronic kidney disease. Critical Care 2015, 19.

4. Sun LY, Wijeysundera DN, Tait GA, Beattie WS: Association of intraoperative hypotension with acute kidney injury after elective noncardiac surgery. Anesthesiology 2015, 123(3):515-523.

5. Walsh M, Devereaux PJ, Garg AX, Kurz A, Turan A, Rodseth RN, Cywinski J, Thabane L, Sessler DI: Relationship between Intraoperative Mean Arterial Pressure and Clinical Outcomes after Noncardiac Surgery: Toward an Empirical Definition of Hypotension. Anesthesiology 2013, 119(3):507-515.

6. D'Alessio JG, Weller RS, Rosenblum M: Activation of the Bezold-Jarisch reflex in the sitting position for shoulder arthroscopy using interscalene block. Anesth Analg 1995, 80(6):1158-1162.

7. Pohl A, Cullen DJ: Cerebral ischemia during shoulder surgery in the upright position: a case series. $J$ Clin Anesth 2005, 17(6):463-469.

8. Koga T, Miyao M, Sato M, Hirota K, Kakuyama M, Tanabe H, Fukuda K: Pituitary apoplexy during general anesthesia in beach chair position for shoulder joint arthroplasty. J Anesth 2010, 24(3):476478.

9. So J, Shin WJ, Shim JH: A cardiovascular collapse occurred in the beach chair position for shoulder arthroscopy under general anesthesia -A case report. Korean J Anesthesiol 2013, 64(3):265-267.

10. Charlson ME, MacKenzie CR, Gold JP, Shires GT: Postoperative changes in serum creatinine. When do they occur and how much is important? Ann Surg 1989, 209(3):328-333.

11. Kim BJ, Lim CS, Hong BH, Lee JY, Lee SY, Lee JU, Kim YH, Lee WH, Yoon SH: Effect of an epinephrine mixture for interscalene block on hemodynamic changes after the beach chair position under general anesthesia: a retrospective study. Korean J Anesthesiol 2017, 70(2):171-176.

12. Loef BG, Epema AH, Smilde TD, Henning RH, Ebels T, Navis G, Stegeman CA: Immediate postoperative renal function deterioration in cardiac surgical patients predicts in-hospital mortality and long-term survival. J Am Soc Nephrol 2005, 16(1):195-200.

13. Kwak HJ, Lee JS, Lee DC, Kim HS, Kim JY: The effect of a sequential compression device on hemodynamics in arthroscopic shoulder surgery using beach-chair position. Arthroscopy 2010, 26(6):729-733.

14. Cho SY, Kim SJ, Jeong CW, Jeong CY, Chung SS, Lee J, Yoo KY: Under general anesthesia arginine vasopressin prevents hypotension but impairs cerebral oxygenation during arthroscopic shoulder surgery in the beach chair position. Anesth Analg 2013, 117(6):1436-1443.

15. Laflam A, Joshi B, Brady K, Yenokyan G, Brown C, Everett A, Selnes O, McFarland E, Hogue CW: Shoulder surgery in the beach chair position is associated with diminished cerebral autoregulation but no differences in postoperative cognition or brain injury biomarker levels compared with supine positioning: the anesthesia patient safety foundation beach chair study. Anesth Analg 2015, 120(1):176-185.

16. Post EH, Vincent JL: Renal autoregulation and blood pressure management in circulatory shock. Crit Care 2018, 22(1):81.

17. Rhee CJ, Kibler KK, Easley RB, Andropoulos DB, Czosnyka M, Smielewski P, Brady KM: Renovascular reactivity measured by near-infrared spectroscopy. J Appl Physiol (1985) 2012, 113(2):307-314. 
18. Correas JM, Anglicheau D, Joly D, Gennisson JL, Tanter M, Helenon O: Ultrasound-based imaging methods of the kidney-recent developments. Kidney Int 2016, 90(6):1199-1210.

\section{Tables}

Due to technical limitations, Tables 1 - 4 are only available for download from the Supplementary Files section.

\section{Figures}

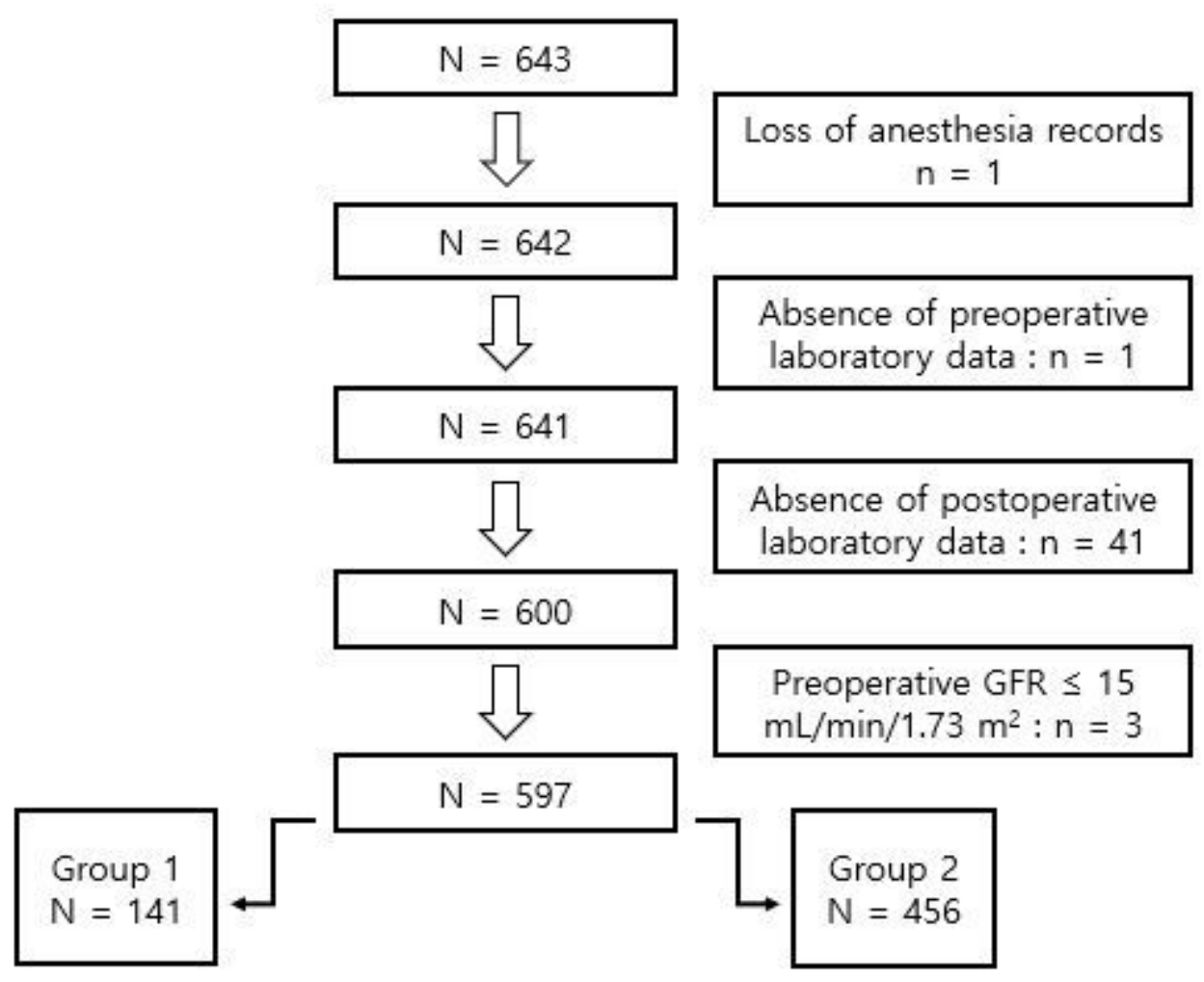

Figure 1

Flow chart of patient enrollment in the study. GFR, glomerular filtration rate. Patients whose MAP fell below $50 \mathrm{mmHg}$ even once were classified into the hypotension group (Group 1), while those in whom blood pressure did not fall below $50 \mathrm{mmHg}$ were classified into the normal group (Group 2)

\section{Supplementary Files}

This is a list of supplementary files associated with this preprint. Click to download.

- Table3.docx

- Table2.docx

- Table4.docx 
- Table1.docx

Page 12/12 